\title{
$[2]$
}

\section{The Aesthetic Realm}

The everyday world is filled with aesthetic experiences, yet anthropology has barely scratched the surface of this most significant area of human behavior. Part of the problem is that the philosophical basis of aesthetics is so shaky. Although philosophical investigations in aesthetics have their origins in antiquity, J. O. Urmson could write not too long ago: "Philosophers have hoed over the plot of aesthetics often enough, but the plants that they have raised thereby are pitifully weak and straggling objects. The time has therefore not yet come for tidying up some corner of the plot; it needs digging over afresh in the hope that some sturdier and more durable produce may arise, even if its health be rather rude" (1957:75). Nor is Urmson alone in his seemingly radical opinion (see, for example, Weitz 1956, 1977; Passmore 1951; Kennick 1958; Aschenbrenner 1959; and Ziff 1984). If after nearly three millennia theory and method in aesthetics are still in the starting blocks, then rational inquiry seems doomed. But these modern dissenters provide the anthropologist with an important lead. Many are inclined to abandon as unanswerable, absurd, meaningless, or trivial the ageold question of what an aesthetic form is and instead inquire into what an aesthetic form does (see Tejera 1965).

I have two relatively modest goals for this book. The first is to document the entire aesthetic sphere of a community in all of its myriad details. The second is to give some account of what the people in the community do with their aesthetic forms. The larger aim is to see how the aesthetics of everyday life works as a system, how aesthetic forms work in harness, and how people manipulate the aesthetic sphere to achieve desired outcomes.

Almost inevitably the first question is the definition of "aesthetic." 
If the first ethnographic task is to document aesthetic forms or experiences, then presumably the fieldworker should be able to delineate the field. But this is precisely the mess the philosophers got themselves into. To attempt a definition of "aesthetic" at the beginning of the investigation is to embark on theory prematurely and inappropriately. Instead of rigorous definitions, all we need at this stage is a set of signposts to point us in the right direction.

Attempts by aestheticians to give rigid definitions of aesthetic form, aesthetic experience, aesthetic genre, art, and so forth have repeatedly failed. (See Maquet 1986, Merriam 1964:259-76, and D’Azevedo 1958 for useful discussions of the philosophical problems as they relate specifically to anthropology. Dickie 1971 and Redfern 1983 provide concise reviews of the philosophical literature.) One half of the problem lies within the very nature of aesthetics: as it is a creative sphere, new forms repeatedly arise to challenge old notions. In technical terms, aesthetic form is not a closed concept-that which may be rigorously delineated by definition or enumeration or both (Weitz 1956). "The sonnets of Shakespeare," for example, is a closed concept bounded either by a set of necessary and sufficient conditions (sonnet form, written by William Shakespeare, and so on) or by simple enumeration (numbers 1-154). Aesthetic form is inherently an open concept and cannot be defined in terms of necessary and sufficient conditions.

The other half of the problem is that definitions generate theory prior to investigation of objects and behaviors. They tend to define aesthetic forms and experiences in terms of emotion, symbolism, referential meaning, expression, creativity, or formal relationships so as firmly to lodge all conclusions of subsequent investigations in the definitions. The charge of circularity is often and accurately aimed at aestheticians.

Morris Weitz has recommended a new program that avoids definition and theory as the first steps (Weitz 1956:176). He follows a line of reasoning proposed by Ludwig Wittgenstein in Philosophical Investigations (1953, pt. 1, secs. 65-77). Wittgenstein argues that the term game (like art) cannot be rigorously defined. When we begin to list all of the games we know, we find it increasingly difficult to find a feature or group of features common to them all which can be used as definitive. Instead:

We see a complicated network of similarities overlapping and criss-crossing: sometimes overall similarities of detail (\# 65). 
I can think of no better expression to characterize these similarities than "family resemblances"; for the various resemblances between members of a family: build, features, colour of eyes, gait, temperament, etc., etc. overlap and criss-cross in the same way. - And I shall say: "games" form a family (\# 67).

Similarly Weitz argues that instead of trying to define art one should attempt to characterize it by first enumerating examples of objects people generally agree are art and then extrapolating from them to those objects about which some doubt exists. From these enumerations may arise empirical generalizations, that is, characteristics that make up the family resemblances in the "art family." These are not universal generalizations, nor are they necessary and sufficient conditions. It is not necessary for an object to have all of them to be designated $a r t$.

This book provides an enumeration of aesthetic forms in a community, so to begin the listing here is unwarranted. It is possible, however, to provide a few preliminary, empirical generalizations concerning aesthetic forms to act as orienting concepts. Aesthetic forms are:

1. Capable of sensory perception.

2. Open to judgments based on taste.

3. Capable of affecting the perceiver.

4. Capable of disinterested appreciation.

At this stage of the investigation these generalizations are rough aids in orientation and may or may not provide the basis of family resemblances later. They are useful because they preserve some of the essential characteristics of aesthetic inquiry from the past three thousand years and therefore conform to relatively common understandings of the meaning of aesthetic. But they do not constitute a rigid definition for determining what is and is not aesthetic. Nor are they complete descriptions of aesthetic forms. Aesthetic forms may have many more features, but these few are sufficient for initial orientation.

Why are these generalizations appropriate starting points? After all, one of the most serious criticisms of the "family resemblances" method asks why one set of resemblances should be preferred to any other (Mandelbaum 1965). What follows is not meant as a review of the history of aesthetics, nor as a full-scale review of the generalizations, which is outside the scope of this work. (Redfern 1983 provides a good general overview of the key issues. For recent critiques of the Wittgensteinian method see Carroll 1986 and Eldridge 1987.) 
The word aesthetic is derived from the Greek adjective aisthetikos, "capable of sensory perception." In Platonic philosophy the distinction between the aestheta, entities perceptible to the senses, and the noeta, entities knowable through the mind, is of paramount importance. In the well-known similes of the Cave, the Sun, and the Divided Line in Books 6 and 7 of the Republic, Plato sharply distinguishes between the physical world and the intelligible world, the former appreciated by the senses and subject to error, the latter appreciated by the mind and free from error. Although since classical times the term aesthetic has been more narrowly defined, "being open to sensory appreciation" is still one of its fundamental semantic components.

In his Primitive Art, Franz Boas includes under the rubric aesthetic forms that appeal to all the senses (1927:9-10). At the outset he claims that "forms that appeal to the eye, sequences of tones and forms of speech which please the ear, [and] impressions that appeal to the senses of smell, taste and touch" may all have aesthetic value.

The word aesthetic took on an additional meaning when Alexander Baumgarten suggested in Reflections on Poetry that "aesthetics" serve as a synonym for "the philosophy of taste" (1735). When added to the old Greek word, this new meaning considerably refined the definition and established a program of inquiry that is alive today. Are aesthetic tastes universal? cultural? idiosyncratic? Are they based on rational principles? Are they quantifiable? Are they reducible to formal properties of aesthetic objects? These questions have been of prime importance in aesthetics since Baumgarten's day.

In this context taste does not refer to idiosyncratic likes and dislikes of individuals alone. It is a complex term. Taste may involve deep critical appraisal that can be shared or may be superficial and personal. The former is closer to the eighteenth-century notion, so that it is appropriate to say that aesthetic forms are open to criticism in all senses of the term.

The debate concerning the nature of aesthetic taste prompted some philosophers to consider aesthetic experiences as based on feelings, or affect, even though these scholars often fundamentally disagreed as to what this assertion implied for the possibility of developing a rational theory of taste (compare, for example, Hutcheson 1725 and Kant 1790). These speculations were taken up by the nineteenth-century Romantics and recast into a familiar theory of aesthetics in which affect is of primary significance (e.g., Arnold 1865, Carlyle 1828, Words- 
worth 1800). The view of aesthetic forms as affecting is found very commonly in anthropology (e.g., Boas 1927, Armstrong 1971, Mills 1957) and frequently used as the starting point of analysis.

Finally, the debate concerning aesthetic tastes also spawned the notion that there is a way of experiencing forms which is uniquely aesthetic and is marked by an attitude of disinterested contemplation. In essence the form is appreciated for its own sake alone and not because of any utility or instrumentality it may have. This approach is commonplace and stands behind such catchphrases as "art for art's sake." One could, for example, view the smooth, hard curve of a wellcrafted chef's knife with enjoyment purely for its own sake and not with respect to its capacity to chop onions efficiently. Such an experience is commonly considered to be aesthetic by philosophers and anthropologists alike (see Maquet 1986, Osborne 1970, Dickie 1971).

This very brief discursus is not meant to assert that my generalizations concerning the term aesthetic have some kind of magical, a priori validity because of historical precedents. My intention is simply to show that they point in the right direction and do not in the process apply some arcane, obscure, or narrowly technical meaning to the concept aesthetic. As I have already argued, it is pointless to begin an analysis of aesthetics by defining the subject matter in an idiosyncratic manner.

These generalizations help us distinguish the aesthetic and the nonaesthetic, and a few examples will be instructive, provided it is borne in mind that this process cannot be conducted mechanically: the generalizations are not necessary and sufficient conditions. New cases may arise that require us to add further generalizations or admit of exceptions. This is the strength of a family resemblances approach.

The taste of raw onions is aesthetic. It is perceptible to the senses, some people like it and others do not, it can arouse pleasurable feelings, and it can be savored without concern for the good or harm the onions are doing the eater. The look of a sunset is aesthetic. It is visible, one may or may not like any or all sunsets, it can be moving, and it can be viewed without concern for meteorological portents and the like. Similarly, the way a garden is laid out, the way a house is painted, the look of a duck decoy, the smell of fried chicken, all are aesthetic.

On the other hand, certain things are clearly not aesthetic. The color of the studs that frame a house is not an aesthetic aspect of the house. The studs are not visible, the color is hardly a matter of taste, 
and it is difficult to conceive of the chosen color arousing feelings through contemplation.

So far the discussion has been rather more philosophical than anthropological and has been cast in terms of the abstract potential of things as opposed to concrete behavior that can be studied ethnographically. This is so because the generalizations are signposts for the ethnographer, indicating possible starting points for observation. As soon as the ethnographer observes people going about their daily lives, however, a new dimension is added. In this context the aesthetic potential of an object may or may not be realized. The look of a sunset can be aesthetic, but farmers in a particular town may never look at a sunset without concerning themselves with what they believe it predicts for the next day's weather. So the ethnographer starts to document what has the potential to be aesthetic experiences but must then decide whether particular experiences are aesthetic or not. This decision necessitates getting to know the subjective states of individuals, which is notoriously difficult (some would say impossible) and is perhaps the single most important reason why aesthetic anthropology has not attracted consistent interest.

A truly anthropological investigation of aesthetics, then, must concern itself both with the range of entities that have the potential to be contemplated aesthetically and with the reasons why some are and some are not. These are natural steps in explaining what the aesthetic experience does for individuals or communities. To take an example from my own fieldwork, fishermen in the community of Tidewater never view their boats in a disinterested fashion. The look of a boat is potentially aesthetic, but that potential is never realized. An understanding of why fishermen choose not to view their boats aesthetically is vital for comprehending their general worldview and gives us a context for those objects, such as duck decoys, which they do view aesthetically. Because of these observed behaviors the ethnographer is justified in concluding that for this community decoys are aesthetic forms whereas boats are not.

It may seem tempting to simplify matters by dispensing with the notion of aesthetic form and concentrating exclusively on aesthetic behavior, because the study seems to depend on the ways in which people relate to entities rather than on qualities inherent in the entities themselves. It is certainly true that entities are not by definition aesthetic and that the generalizations regarding the aesthetic status of entities concern how people may relate to these entities. But aesthetic 
forms are still aesthetic forms when they are not being viewed aesthetically, and people manipulate them for a variety of ends (aesthetic and nonaesthetic) knowing they are aesthetic forms even though the act of manipulation is not an aesthetic experience. Hanging a painting in a room may involve functional considerations such that in the process of hanging, the painting is not viewed aesthetically at all. But it is being hung with the full knowledge it is an aesthetic object that will be viewed aesthetically at some later date. The person hanging the painting is responding to its potential as an aesthetic object and not directly experiencing it in aesthetic terms. Thus an exclusive focus on aesthetic experiences confines ethnographic investigation too narrowly. To discover when people have the opportunity for aesthetic experiences but decline to take it can be enlightening and enrich our understanding of aesthetic choices. Such analysis is possible only if we begin by asserting that some forms have the potential to be viewed aesthetically, that is, they are aesthetic forms.

I have deliberately chosen to work in aesthetics rather than art for several reasons. The terms are often confused in anthropological, philosophical, and popular writing, but they can be distinguished, thereby clarifying discussion considerably. Art work is a subset of the category aesthetic form: all art works are aesthetic forms but not all aesthetic forms are art works. An art work is an object or form whose primary (or sole) purpose is to be contemplated aesthetically (see Carroll 1986 for a discussion of the philosophical issues involved). Some works-a sculpture, painting, sonata, play-are art because they were consciously created as such, and they may be called "art by destination" (Malraux 1967). Other works are art even though they were not expressly created as such because they have been placed in a context that forces or encourages a viewer or audience to overlook their original purpose and instead contemplate them aesthetically. Such works may be called "art by metamorphosis" (Malraux 1967). A typewriter in a glass case in a museum is art by metamorphosis. Although the machine may type perfectly well, the museumgoer must ignore that function and derive pleasure by dispassionately observing line, form, color, and so forth. Whether a work is art by destination or art by metamorphosis, we in the West recognize it as art by a number of means, including the way in which it is presented or framed. A sculpture may be framed by a glass case, a classical sonata is framed by stylized behaviors such as tuning, bows, and applause. Museums and concert halls are not everyday fare for most Westerners, however, so that to define aesthetic anthropology in terms of art is to narrow the 
scope of investigation to the point of excluding the majority of aesthetic experiences.

A considerable number of anthropological investigations into aesthetics, like their philosophical counterparts, have tended to focus on art and have concerned themselves with questions of framing, presentation, criticism, and the like (see, for example, Boas 1927, Fischer 1961, Otten 1971, Jopling 1971). The problem with this approach, as many anthropologists have noted, is that the category art meaning "work whose primary purpose is to be contemplated aesthetically" seems to be a predominantly Western concept (see Maquet 1986, D'Azevedo 1973). What is called "primitive art" or "non-Western art" in the anthropological literature are forms that do not function exclusively or even primarily as aesthetic entities. A Balinese mask may owe its form as much to ritual, religious, or magical considerations as to aesthetic concerns. Are we justified in calling the mask "art"? Jacques Maquet (1986) has explored this problem at length, and he concludes that the term "art" is justified because it is possible to identify in many non-Western cultures behaviors that are analogous to what we in the West consider important for defining an art work, namely, appropriate framing, criticism based on good form, disinterested contemplation, and so on. I am nonetheless concerned that a focus on art limits the sphere of study and discourse.

Even in the West, art works are a very small subset of what I call aesthetic forms. To limit analysis to art is to claim that in some way one kind of aesthetic experience is special. This potentially elitist argument has brought some anthropologists to argue for the expansion of the term art to include a much wider variety of aesthetic forms. Boas, for example, states that a "composition of scents, a gastronomical repast may be called works of art provided they excite pleasurable sensations" (1927:10), although he adds the following to expand his definition: "When the technical treatment has attained a certain standard of excellence, when the control of the processes involved is such that certain typical forms are produced, we call the process an art, and however simple the forms may be, they may be judged from the point of view of formal perfection; industrial pursuits such as cutting, carving, moulding, weaving; as well as singing, dancing and cooking are capable of attaining technical excellence and fixed forms" (1927:10). Such a broadening of the definition of art has a clear appeal for those troubled by the potentially elitist connotations of the term. After all, the word carries with it notions of approval, so that to call a work "art" is to give it value in a prestigious sphere. Quilts, duck decoys, dance 
masks, decorated pots can be called "folk art" or "primitive art" and thereby, at least nominally, accorded the rights and privileges of a Matisse (that is, laying aside the condescension inherent in the terms "primitive" and "folk"). Though I am sympathetic to this social cause, I believe the confusion that results from redefining art seriously impedes analysis of aesthetics.

A pot used for carrying water may be highly decorated, but no amount of aesthetic elaboration can alter the fact that the pot is an object with a utilitarian purpose. As such it is fundamentally different from a ceramic sculpture that has no utilitarian purpose. In the interest of accurate ethnographic reportage, if for no other reason, we ought to keep the categories distinct. This distinction does not summarily exclude quilts and duck decoys from the category art. It is quite conceivable (see Forrest 1983) that duck decoys are made and used almost exclusively as objects of aesthetic appreciation and deserve to be called "art" in the conventional sense. Their supposed utility may screen their basic aesthetic purpose, but only careful ethnographic observation will reveal the fact.

Beyond the need to report one's fieldwork experiences precisely is a more obvious reason for not expanding the term art endlessly. Although few if any scholars would recommend the notion, the term art could hypothetically be expanded to include all aesthetic forms, certainly solving the problem of elitism but making two terms serve the same function and leaving unmarked the category of art for art's sake. This redefinition also entails the inclusion under the rubric art of sunsets, storms at sea, bird songs, the smell of a rose; most analysts for a variety of theoretical reasons want to exclude natural phenomena from consideration as art (not least because art and artifact are closely related semantically and etymologically). By omitting natural phenomena from the definition, however, the observer either ignores an important set of aesthetic experiences or else documents all aesthetic experiences but arbitrarily distinguishes between those which are art and those which are not. In the first case important data are lost, and in the second the observer ends up sorting field data into two piles because of preconceived categories that may or may not have any local meaning. Any other expansion or contraction of the term art runs the same risk.

The simple solution, which I have adopted, is not to think in terms of art at all. Freed from categorical assumptions, I claim the widest possible observation of aesthetic forms and experiences. Such an approach might well be adopted throughout aesthetic anthropology. Af- 


\section{Lord I'm Coming Home}

ter all, many ethnographers are interested in aesthetics rather than art and waste a good deal of theoretical energy justifying their subject matter as art when what they really want to discuss are good form, critical perspectives, disinterested contemplation, technical complexity, creativity - all of which can be discussed adequately without reference to art.

Another problem is the tendency to think in terms of conventional Western art genres, such as music, dance, drama, sculpture, and poetry, and to specialize in the analysis of one only. Such specialization entails an unfortunate limitation. To undertake a cross-cultural analysis of music, for instance, requires considerable technical sophistication. The fieldworker must have comprehensive ear training, a thorough grounding in general music theory, and a deep understanding of and sensitivity to musical forms unusual in Western music, such as microtones, polyrhythms, and gapped scales. In addition, recording and documentation require special training, all of this to be absorbed on top of general anthropological theory and method. Given such stringent requirements, ethnomusicology has produced many penetrating insights into aesthetic behavior (for an overview see Merriam 1964). However, these insights come at a cost.

There is no reason to suppose that the aesthetics of music is an isolated cultural category. Even in the West music is inextricably linked to language (in song) and movement (in dance), and it may serve as a frame or background for a variety of aesthetic and nonaesthetic activities. A focus on music alone may miss important associations between aesthetic forms (see Brenneis 1987). Similar problems arise in any single-genre analysis.

A partial solution can be found in a multigenre approach, as Boas proposed in Primitive Art (1927). Such studies are rare but have been attempted periodically (Armstrong 1971, Price and Price 1980, Price 1984). Even multigenre approaches, no matter how catholic, miss some of the aesthetic forms and experiences in the community under study. I know of none that deals with the aesthetics of the natural environment, for example. Yet how carvers view living plants may be of fundamental importance to how they carve plants. In short, I believe that nothing less than a survey of the entire aesthetic realm of a community can provide an aesthetic context comprehensive enough for the situating of individual aesthetic experiences.

No one has attempted an ethnography of the entire aesthetic realm of a community, although many may have contemplated it. The task is made immensely difficult by compounding problems. First, the aes- 
thetic realm has resisted attempts to delimit and conceptualize it adequately. Second, the range of aesthetic experiences in even a small community is potentially enormous. The sheer size of the aesthetic realm presents the fieldworker with a hard task even without other theoretical or methodological complications. The diversity of the materials requires the fieldworker to master a whole battery of notation and documentation techniques and to be sensitive to nuances in the forms of entities as different as fried fish and the tango. Yet it is precisely because the aesthetic realm is so vast and omnipresent that it ought to be a major concern in anthropology.

Warren D'Azevedo gives a summary history of aesthetic anthropology in The Traditional Artist in African Societies (1973:1-14). Here I want to point out two approaches that have received wide attention and that bear on the methods I employed: quantitative investigations and "symbolic" studies.

Quantitative investigations into aesthetic phenomena were for a time very popular and promised significant insights into the relationships between aesthetic value and social structure. Much of the impetus for this research came from the culture and personality school-anthropologists concerned with the interplay between individual psychology and the culture the individuals were raised in.

Herbert Barry (1957) sought statistically rigorous relationships between the formal characteristics of "works of graphic art" from thirty societies and the severity of socialization of children in those societies as measured on a scale developed by John Whiting and Irvin Child (1953). Barry took eleven "art variables" such as "presence of sharp figures," "presence of curved lines," and "representativeness of design" and graded ten objects from each society on a seven-point scale for each variable. He formally compared these results with the Whiting and Child scale and tested for statistically significant correlations using standard methods. His main finding was a strong positive correlation between complexity of design and severity of socialization. Other studies followed Barry's lead (Fischer 1961, Lomax 1968), finding a variety of correlations in many media. Fischer, for example, tested a host of social variables, such as degree of egalitarianism, male solidarity in residence, marriage types, against a list of design variables similar to Barry's. By broadening the social variables he was able to find many more correlations. Lomax and his coworkers have done the same for music and dance.

These studies are suggestive, providing fieldworkers with new avenues to explore. However, their results do not appear to serve the 
primary needs of inquiry into the nature of aesthetics, because the methodology used to develop codable variables ends up eliminating from study what is aesthetic about aesthetic forms. The variables must of necessity deal with quantifiable, scalable data, which means choosing objectifiable, formal aspects of objects for analysis. Two cultures will be assessed as similar if selected objects from both show roughly the same number of straight lines. The affective states created by these lines, their local interpretation and criticism, and their relationship to other design elements are not considered. Thus, fundamental aesthetic qualities of the lines are ignored. What is more, the investigators tend to use museum objects, commercial recordings, and the like to build a database without concern for the status of the entities as aesthetic forms in their local environment. A mask is "art" because it is treated as such by a museum, whatever its function in the culture of origin.

The basic problem with these approaches is that aesthetic forms are by nature subject to lack of agreement. This lack of agreement is the very heart of criticism that is fundamental to aesthetic forms. Quantitative studies either study those aspects of aesthetic forms which are indisputable, "this line is straight" - in which case they are not studying aesthetics because the matter is not open to judgment-or else they are studying aspects upon which people disagree-in which case they have no business quantifying them.

Lack of agreement in different cultures (rather than elements of design) has received very little attention in quantitative studies. Irvin Child and Leon Siroto (1965) opened this field by asking members of BaKwele society and art experts in New Haven to rate thirty-nine BaKwele masks. The results were not fully conclusive but indicated that although the general ratings of the two cultures did not agree, the ratings of experts did. Previous psychological studies had failed to show such agreement because they had not isolated the responses of expert critics (Lawlor 1955, McElroy 1952).

This procedure may prove useful in directing the attention of fieldworkers, but I am concerned about the general validity of its elicitation methods. If an informant is looking at an aesthetic form for the purposes of a survey (and perhaps being paid for services rendered), can it be said that he or she is viewing the form in a disinterested manner? Such experiments may prevent the informant from engaging in aesthetic behavior or may elicit responses according to predefined notions of aesthetic criticism. I take this issue up again when dealing with my own fieldwork procedures. 
The second set of approaches to aesthetic materials can be styled "symbolic," although no single rubric can encompass the range of studies involved without oversimplifying. Both James Peacock (1975: 129-153) and Hugh Duncan (1969:47-98) provide penetrating insights into the use of symbolic theory in the anthropological analysis of aesthetics.

It is commonplace to hear aesthetic forms referred to as "forms of expression" or "modes of communication" and to leap from these (controversial) assumptions to think of aesthetic forms as symbolic. By this analysis, aesthetic forms are a language that requires only the appropriate dictionaries and grammar books for adequate translation.

One line of action begins by identifying the units of the aesthetic language under consideration and then examines the structures into which they fit. It follows a well-established mode of linguistic analysis which begins by establishing units of sound (phonemes) and meaning (morphemes) through some form of contrastive opposition and then examines the structures that can be built from them (see Hockett 1958). Alan Dundes (1964), for example, breaks folktales into minimal narrative units (motifemes) and shows how these units are built up into logical narrative structures. Similar methods may be found in the investigation of dance (Kaeppler 1967), artifacts (Deetz 1967), and architecture (Glassie 1975, Preziosi 1979). General systems of signs or symbols have been explored at great length by Claude Lévi-Strauss (1963, 1966, 1969), Roland Barthes (1967, 1972), Edmund Leach (1976), and Dan Sperber (1975), and these works frequently allude to aesthetic forms. The theoretical power and shortcomings of these models are well-known. Indeed, in the final chapter of this book I provide a structural analysis that superficially shares features with the works of these scholars. The points of divergence are of fundamental importance, however, though at this stage I simply give some general guidelines to mark my position.

There is no question aesthetic forms may act as symbols, and when they do so the perspectives of the semiotician and semiologist can provide many insights (see Maquet 1986:93-117 for an outline of the semiotic position on aesthetics). But treating aesthetic forms as signs, icons, or symbols exclusively may limit interpretation in several ways.

A symbol, by definition, stands for something else. Conventionally a symbol is called a signifier and what it stands for, the signified (see Leach 1976 on terminology). Clearly, however, an aesthetic form need not have a signified. As Merriam (1964:229-58) points out, it is difficult to see how most music in the Western tradition can be said to 
signify anything. Music may evoke various affective states without signifying them (which would not necessarily evoke them, anyway) and without signifying other objects that would evoke them. Also, some categories of aesthetic forms, natural objects for example, are not symbols until deliberately cast as such. And even if an aesthetic form does have symbolic aspects or as a whole may be treated as a symbol, the recognition of assorted signifieds does not provide an exhaustive understanding of the form because the form can still be appreciated without consideration of the signifieds. The Statue of Liberty is burdened with multilayered symbolism, but it can still be viewed in isolation from all these symbols.

Treating aesthetic forms as symbols, and especially treating them as languagelike, presupposes that they stand as intermediaries between two communicators. As Armstrong (1971) has amply demonstrated, however, we have no reason to think of aesthetic forms as conveyors of meaning from one person to another: we may respond directly to them and need not try to understand what they communicate from someone else. It is possible to establish a relationship directly with an aesthetic form without wondering what another person is saying through the form. Again, this point is obvious in the case of natural phenomena, and one can extend one's experience from these to other aesthetic forms.

Those who take a symbolic approach to aesthetic forms find it hard to break away from a content analysis because symbolic interpretation often entails looking for signified or referential meaning - that is, content. But aesthetic forms have form, and their form is of vital importance (see Alland 1977, 1983). Some scholars, notably Lévi-Strauss, overlook form when convenient: his analysis of the Oedipus story is a classic example (1963:206-231). Even when symbolic form is given some attention, it may still be treated as a vehicle for communication and not as an aspect of an object worthy of discussion without respect to signification. Rather than conveying meaning, however, aesthetic form may be identical with its meaning. The harmonious juxtaposition of shapes in a painting does not simply represent order, it is order.

The basic idea in philosophy that a work can be divided into its form (relationships of elements in the medium employed) and content (what it represents) can be traced to Plato and found detailed expression in the aesthetic treatises of Kant (1790). Thus a painting, by this analysis, may have aspects of form (use of color, arrangements of shapes, surface texture) and content (the Crucifixion, the Birth of Venus). 
Formalists took the form/content distinction to be vital in understanding a work aesthetically, arguing that aesthetic judgments appropriately lay in the appraisal of form alone; content was not relevant. Roger Fry (1920), for example, speaks of "mediumistic" values as those suitable for discussion in aesthetic terms. This position (see, for example, Bell 1914, Parker 1926, Eichenbaum 1926) was responsible in large measure for the later development of several schools of structuralism.

Many philosophers have nonetheless been uncomfortable with the form/content distinction (see, for example, Weitz 1950, Ransom 1941, Brooks 1947, Blackmur 1952, Tate 1968, Kaplan 1954, and Vivas and Krieger 1953). First, the distinction is not applicable to all works. It makes some sense when applied to figurative painting, literature, and the like but has little or no value when discussing music or smells. Second, there is no analytic need to divide aspects of a work in this fashion, and to do so often involves wrangling over strict definitions. It is much simpler to talk of various aspects, such as shape, size, feel, color, loudness, hardness, physical composition, referential meaning, and iconic references. (It is still occasionally convenient to refer to manipulation of the medium in terms of "formal" qualities, but the reference is now simply shorthand and not a strictly defined analytic term.)

The fact that the form/content distinction does not work in aesthetics is an important reason for keeping aesthetic and symbolic analysis distinct. A symbol has, by definition, a form and a content. Without content it is not a symbol, and much symbolic analysis rests on preserving the form/content distinction (for an informative debate see Morris 1939 and Price 1953).

I advocate, therefore, that we treat aesthetic forms as aesthetic forms. Quantitative and symbolic methods have been extremely powerful, but their use can direct attention away from aesthetics as such. My aim here is primarily to serve the needs of aesthetic forms and to search for bridges to other methods of analysis only when necessary to deepen the understanding of aesthetics. 